\title{
Analysis of the Behavior of a Chaotic Dynamic System under Different Types of Couplings and Several free Dynamics
}

\author{
Clara Grácio ${ }^{1}$, Sara Fernandes ${ }^{1}$, Luís Lopes ${ }^{2}$, Gyan Bahadur Thapa ${ }^{3}$ \\ ${ }^{1}$ School of Science and Technology, Universidade de Évora, Research centre CIMA-UE, Portugal \\ ${ }^{2}$ Instituto Superior de Engenharia de Lisboa, Portugal \\ ${ }^{3}$ Department of Applied Sciences, Pulchowk Campus, Institute of Engineering \\ Tribhuvan University, Nepal \\ Corresponding author: ${ }^{1}$ saf@uevora.pt, ${ }^{1}$ mgracio@uevora.pt, ${ }^{2}$ luismariolopes@gmail.com, \\ ${ }^{3}$ thapagbt@ioe.edu.np
}

Received: Dec 31, 2018

Revised: March 27, 2019

Accepted: April 2, 2019

\begin{abstract}
In this paper, we analyze how the behavior of a chaotic dynamical system changes when we couple it with another. We focus our attention on two aspects: the possibility of chaos suppression and the possibility of synchronization. We consider a Symmetric Linear Coupling and several free dynamics. For each of them we study the evolution of the coupling behavior with the coupling strength constant, defining windows of behavior. We extend the analysis to some other couplings. This is a survey paper.
\end{abstract}

Key words: chaotic dynamical systems, symmetric linear coupling, suppression of chaos, synchronization

\section{Introduction}

In our world, there is nothing isolated, whether it be an atom or a constellation, a person or a computer, information or matter, a cell or a tornado. The existence of interaction between dynamical systems can substantially alter certain characteristics of their behavior, so the study of the coupling of dynamical systems is particularly relevant. Two issues are particularly important: the possibility of the chaotic behavior of a dynamical system be suppressed when the dynamical system is coupled to another and the possibility of synchronization [10] between the two systems. The study of couplings, in addition to making it possible to identify and clarify some of the main issues arising from "not-being-alone", opens the door to the analysis of the immense world of networks.

For the study of behaviors, we use an analytical approach and also a numerical approach. In Section 2, we expose some generalities regarding dynamical systems relevant to the foray into 
the suppression of chaos and synchronization that we make in the following section. With regard to these generalities, we present the dynamics we use to exemplify the results we obtain throughout the work. Bearing in mind that we want the emphasis to be placed on the clarification of questions that the existence of a coupling can cause and since such questions are to a large extent independent of certain characteristics of the chaotic dynamical systems that we use, we have chosen to consider only discrete, one-dimensional and autonomous dynamical systems. Most of the issues raised can all the same be treated using the type of coupling that the literature most often uses and which we refer to as the Symmetric Linear Coupling [3, 5, 7, 9, 12, 11]. In Section 3, Symmetrical Linear Coupling is defined and analyzed suppression of chaos and synchronization that may result from this coupling [8]. We also extend them to some other couplings.

\section{Chaotic Dynamical Systems}

\subsection{Dynamical Systems}

Consider a dynamical system $(x, T, M)$, with $x: T \times M \rightarrow M$. This system is said to be discrete when $T=\mathrm{IN}$, i.e. when the evolution parameter $t$ is a natural number. The fact that a dynamical system is one-dimensional means the phase space $M$ is the set of real numbers or a subset of it, $I$, the iteration set. We will consider only autonomous dynamical systems, i.e. dynamical systems whose description of evolution does not involve explicit dependence on the parameter $t$. Such dynamical systems can be described by applications, i. e. by functions $f: I \rightarrow I$, since the set of all iterations $\mathrm{x}(t)$, with $t \in \mathrm{IN}$, corresponding to an initial iteration $x_{0}$, can be given by the solution of the differences equation

$$
\left\{\begin{array}{l}
x(t+1)=f(x(t)) \\
x(0)=x_{0}
\end{array}\right.
$$

It is said that two dynamical systems are identical if they are described by the same application. Establishing connections between $N$ one-dimensional dynamical systems, $x_{1}, x_{2}, \ldots, x_{N}$, i.e. creating a network of one-dimensional dynamical systems, gives rise to a dynamical system with $M=I^{N}$ described by a function $\vec{f}: I^{N} \rightarrow I^{N}$.

$$
\left\{\begin{array}{l}
\vec{x}(t+1)=\vec{f}(\vec{x}(t)) \\
\vec{x}(0)=\vec{x}_{0} .
\end{array}\right.
$$

The coupling of two one-dimensional dynamical systems corresponds to the particular situation where $N=2$. The set of all iterations associated with an initial iteration $\vec{x}_{0}$ defines a trajectory that is designated by $\left(\vec{x}_{0}, \vec{x}(1), \vec{x}(2), \ldots, \vec{x}(t), \ldots\right)$, or, more abbreviated, by $(\vec{x}(t))$. Some initial iterations $\vec{x}_{0}$, namely the solutions of $\vec{x}_{0}=\overrightarrow{f^{(n)}}\left(\vec{x}_{0}\right)$, with $\overrightarrow{f^{(n)}}=\vec{f} \bullet \vec{f} \bullet \ldots \bullet \vec{f}$ ( $n \in$ IN times), 
give rise to trajectories that verify equality $\vec{x}(t+n)=\vec{x}(t), \forall t \in \mathrm{IN}$. These trajectories are called periodic and are completely described by the enumeration of $n$ first iterations, $\left(\vec{x}_{0}, \vec{x}(1), \vec{x}(2), \ldots, \vec{x}(n-1)\right)$. The case where $n=1$ corresponds to the fixed-point situation, $\vec{x}(t)=\vec{x}_{0}$. If initial iterates near a certain trajectory $(\vec{s}(\mathrm{t}))$ give rise to trajectories that, for $t$ large enough, approximate to $(\vec{s}(\mathrm{t}))$ as much as we want, then $(\vec{s}(\mathrm{t}))$ is said to be an attractive trajectory. An exponentially stable solution of $\vec{x}(t+1)=\vec{f}(\vec{x}(t))$ is an attractive trajectory.

Definition 1: It is said that $(\vec{s}(t))$ is an exponentially stable solution (or trajectory) of $\vec{x}(t+$ 1) $=\vec{f}(\vec{x}(t))$ if there exist $a, b, \delta, \in R+$ such that, if $\|\vec{x}(0)-\vec{s}(0)\|<\delta$, then

$$
\|\vec{x}(t)-\vec{s}(t)\| \leq a\|\vec{x}(0)-\vec{s}(0)\| . e^{-b t}, \forall t>0 .
$$

Definition 2: The basin of attraction of a solution $(\vec{s}(t))$ exponentially stable solution of $\vec{x}(t+$ $1=f(x t)$ is the set of initial iterates $x 0$ for which $\lim t \rightarrow+\infty / / x t-s t / /=0$. The immediate attraction basin of $(\vec{s}(\mathrm{t}))$ is the subset of the attraction basin for which all the separated parts that compose it comprise at least an iteration $\vec{s}(t)$.

\subsection{Chaotic Behavior}

When a trajectory does not approach a periodic one, we can be dealing with a dynamical system with chaotic behavior. The study of chaos is relatively recent, so there are still several alternative definitions of chaotic behavior, not all equivalent [1], [2], [6]. In 1989, Devaney defined that a dynamical system $\vec{x}$ described by the application $\overrightarrow{f:} I^{N} \rightarrow I^{N}$ has chaotic behavior if there exists a set $K \subset I^{N}$ such that $\vec{f}$ satisfies the following conditions [4]: 1-- $\vec{f}$ has sensitive dependence on the initial iterate, i.e. there exists a $\delta>0$ such which, for any $\vec{x}_{01} \in K$ and any neighborhood of it, there is a $\vec{x}_{02}$ in that neighborhood and a value $t \in I N$ such that $\left\|\vec{x}\left(t, \vec{x}_{01}\right)-\vec{x}\left(t, \vec{x}_{02}\right)\right\|>\delta .2$ - the set of periodic trajectories is dense in $K .3-\vec{f}$ is topologically transitive in $K$, i.e. for each pair of open sets $U, V \subset K$ there exists a $t \in \mathrm{IN}$ such that $\vec{x}(\mathrm{t}, \mathrm{U}) \cap V \neq \emptyset$. It is common to all definitions of chaotic behavior, the notion that such a behavior determines a sensitive dependence on the initial iteration, which entails a "spreading" of trajectories. This dependence can be quantified by the Lyapunov exponents that account for the increase of the distance between neighboring trajectories. Since we are considering one-dimensional dynamical systems, when these systems are described by sectionally differentiable maps, the expression of their unique Lypaunov exponent can be reduced to

$$
\mu=\lim _{T \rightarrow+\infty} \frac{1}{T} \sum_{t=0}^{T-1} \log \left|f^{\prime}(x(t))\right|
$$

A dynamical system with a positive Lyapunov exponent has a sensitive dependence on the initial iteration. In fact, if the iteration set is limited, the "spreading" of the trajectories that a positive 
Lyapunov exponent determines, imposes a behavior that we identify as being chaotic. In this paper we use a limited iteration set, so we adopted the following definition of chaotic behavior.

Definition 3: If IN $\subset I R^{N}$ is a limited set, it is said that a dynamical system $\vec{x}$ described by $\vec{f}: I^{N}$ $\rightarrow I^{N}$ has a chaotic behavior if at least one of its Lyapunov exponents is positive. In these circumstances it is also said that $\vec{f}$ is a chaotic map.

The maps that we use in the following section are unidimensional continuous maps, with iteration interval $I=[0,1]$ and that present chaotic behavior, namely the ones we call tent, logistic, saw and cubic maps.

(i) The tent map, is defined by,

$$
f_{T}(u)=\left\{\begin{array}{cc}
2 u, & u \in\left[0, \frac{1}{2}[\right. \\
2-2 u, & u \in\left[\frac{1}{2}, 1\right]
\end{array}\right.
$$

It has chaotic behavior, since its value of Lyapunov exponent is $\mu_{T}=\ln 2$.

(ii) If we take the polynomial interpolation obtained using the extreme points of the graph of $f_{T}$ the points $(0,0),\left(\frac{1}{2}, 1\right),(1,0)$ - as nodes, we obtain:

$$
f_{L}(u)=4 u(1-u)
$$

It is the logistic map, $f(u)=r u(1-u)$, with $r=4$.

Since $f_{L} \circ h=h \circ f_{T}$, with $h(u)=\operatorname{sen}^{2}\left(\frac{\pi u}{2}\right)$, the maps $f_{T}$ and $f_{L}$ are topologically conjugated.

That determines that $f_{L}$ and $f_{T}$ have the same Lyapunov exponent. So, the logistic map also has chaotic behavior.

Considering that $f_{T}$ and $f_{L}$ are both unimodal maps, we also wanted to use other maps that are not unimodal ones. We chose two that are related to each other in the same way as $f_{T}$ and $f_{L}$ are, i.e. the saw map, $f_{S}$, is a sectional linear, continuous map, such as $f_{T}$, and the cubic map, $f_{C}$, is the polynomial interpolation that is obtained using the extreme points of $f_{S}$ as nodes.

(iii) Let us define the saw map, $\mathrm{f}_{\mathrm{S}}$, a map with $f_{S}(1)=1$ and such that the image of the referred polynomial interpolation is contained in $I$, i.e. $f_{C}(I) \subset I$, by:

$$
f_{S}(u)=\left\{\begin{array}{cc}
2.4 u, & u \in\left[0, u_{1}\right] \\
1.7-2.4 u, & u \in\left[u_{1}, u_{2}\right] \\
2.4 u-1.4, & u \in\left[u_{2}, 1\right] .
\end{array}\right.
$$


with $u_{1}=\frac{17}{48}$ and $u_{2}=\frac{31}{48}$. The exponent of Lyapunov of $f$ is $\mu_{S}=\ln 2.4$, a positive value, corresponding to the desired chaotic behavior.

(iv) So, we define the cubic map by,

$$
\begin{gathered}
f_{C}(u)=\frac{u\left(u-u_{2}\right)(u-1)}{u_{1}\left(u_{1}-u_{2}\right)\left(u_{1}-1\right)} \cdot 0.85+\frac{u\left(u-u_{1}\right)(u-1)}{u_{2}\left(u_{2}-u_{1}\right)\left(u_{2}-1\right)} \cdot 0.15 \\
+\frac{u\left(u-u_{1}\right)\left(u-u_{2}\right)}{\left(1-u_{1}\right)\left(1-u_{2}\right)}
\end{gathered}
$$

Calculating numerically the Lyapunov exponent of $f_{C}$, using an initial random condition and $10^{7}$ iterates, we obtain $\mu_{C} \simeq 0.715$, a positive value to which also corresponds the intended chaotic behavior of $f_{C}$. These maps have the following graphs:

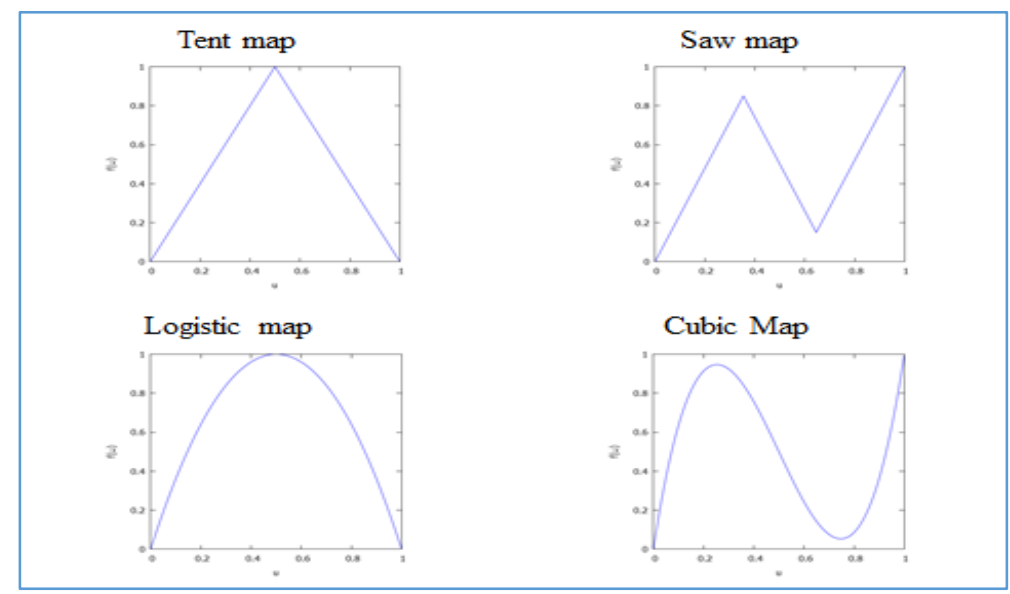

Fig. 1: Graphics of the tent, logistic, saw and cubic maps

\section{Chaotic Dynamical System Couplings}

We analyze in this section the coupling of discrete, unidimensional and autonomous dynamical systems with chaotic behavior. This coupling can be defined by

$$
\left\{\begin{array}{l}
x(t+1)=f(x(t))+F(x(t), y(t), t) \\
y(t+1)=g(y(t))+G(x(t), y(t), t)
\end{array}\right.
$$

where $F, G: \mathrm{R}^{3} \rightarrow \mathrm{R}$ are the interaction functions, and the terms $F(x(t), y(t), t)$ and $G(x(t), y(t), t)$ give an account of the interaction which is established between the two dynamical systems. 
Each choice of $F$ and $G$ corresponds to a different coupling. For its study it is useful to introduce a parameter, the coupling constant, that allows us to control, in a linear way, the extent of the interaction between the coupled dynamical systems:

$$
\left\{\begin{array}{l}
x(t+1)=f(x(t))+c \cdot F(x(t), y(t), t) \\
y(t+1)=g(y(t))+c \cdot G(x(t), y(t), t)
\end{array}\right.
$$

It can be considered, without loss of generality, that $c \in[0,1]$.

Definition 4: We designate by coupling- $c_{0}$ (of the dynamic systems described by maps $f$ and $g$ ) the particularization of (3.2) corresponding to the coupling constant $c_{0}$.

Definition 5: We designate by coupling (of the dynamic systems described by maps $f$ and $g$ ), the set of all coupling- $C_{0}$ defined by (3.2) that a given choice of $F$ and $G$ determines.

If the interactions are autonomous and decomposable in relation to each one of the dynamical systems $x$ and $y$, i.e. if we consider interactions $F(u, v, t)=F_{1}(u)+F_{2}(v)$ and $G(u, v, t)=G_{1}(u)+G_{2}(v)$, we obtain

$$
\left\{\begin{array}{l}
x(t+1)=f(x(t))+c \cdot\left[F_{1}(x(t))+F_{2}(y(t))\right] \\
y(t+1)=g(y(t))+c \cdot\left[G_{1}(x(t))+G_{2}(y(t))\right]
\end{array}\right.
$$

Further, if we consider that the next iteration of each of the coupled dynamical systems results from replacing a fraction of what would be the next-iteration if they were free from each other by what the other free dynamical system would impose, and if that fraction is measured by the coupling constant $c$, we obtain the following coupling

$$
\left\{\begin{array}{l}
x(t+1)=(1-c) f(x(t))+c \cdot g(y(t)) \\
y(t+1)=c \cdot f(x(t))+(1-c) \cdot g(y(t))
\end{array}\right.
$$

This form corresponds to the choice $G_{1}=-F_{1}=f$ and $F_{2}=-G_{2}=g$.

Definition 6: The coupling defined in (3.4) is called Linear Coupling.

In a linear coupling, the interaction terms depend linearly on the maps describing the free dynamics of the coupled dynamical systems and it should be noted that the iterations do not leave the iteration interval.

\subsection{Symmetrical Linear Coupling}

We consider the relevant case where the linear coupling is performed between two identical dynamic systems. This coupling is defined by, 


$$
\left\{\begin{array}{l}
x(t+1)=(1-c) f(x(t))+c \cdot f(y(t)) \\
y(t+1)=c \cdot f(x(t))+(1-c) \cdot f(y(t))
\end{array}\right.
$$

Definition 7: The coupling defined in (3.5) is called Symmetrical Linear Coupling.

\subsubsection{Numerical Approach with Logistic Map}

We begin by choosing that the dynamic of free dynamical systems is described by the logistic map, i. e., $f$ is assumed to be the logistic map. In order to have an overview of the behavior of the coupling after the transient regime, we consider the following numerical approach: we compute the iterations $x(t)$ and $y(t)$ for sufficiently large values of $t$, namely between $t=100$ and $t=200$, using random initial iterates and we use different initial random iterations for each of the values of $c$ considered $(c=i / 1000$, with $i=0,1, \ldots, 1000)$. The three-dimensional graph of the iterations $(x(t), y(t))$ is constructed as a function of $c$, which is shown in the Fig. 2.

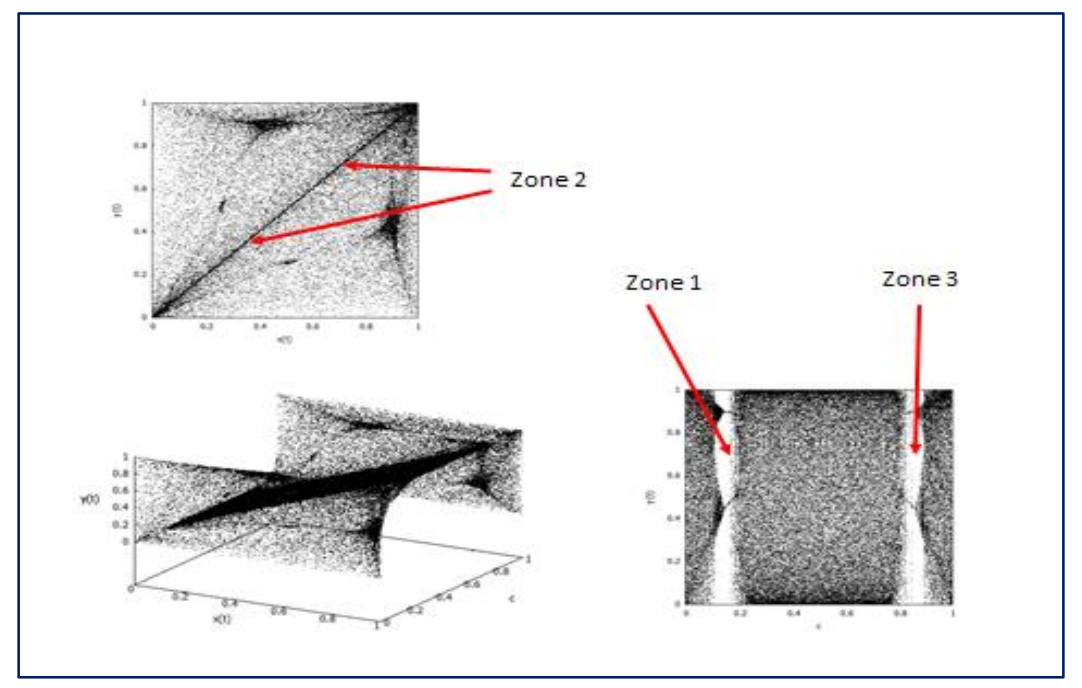

Fig. 2: Graph of $(x(t), y(t))$ as a function of $c$ (bottom left) for Symmetric Linear Coupling of logistic, presenting perspectives of $y(t)$ as a function of $x(t)$ (above) and $y(t)$ as a function of $x(t)$ (bottom right)

The graph identifies three zones in which coupling establish a relation between dynamical systems:

zone 1 - for values of $c$ in a set that includes $c \simeq 0.15$ and values near this one, the behavior of dynamical systems ceases to be chaotic.

zone 2 - for values of $c$ in a set that includes $c \simeq 0.5$ and values near this one, the behavior of dynamical systems remains chaotic but $\mathrm{x}(t)=y(t), \forall t$, which is responsible for the diagonal $y=$ $x$ being highlighted in the perspective that presents $y(t)$ as a function of $x(t)$. 
zone 3 - for values of $c$ in a set that includes $c \simeq 0.85$ and values near this one, the behavior of dynamical systems ceases to be chaotic.

These three important zones correspond to different behaviors that need to be analyzed in detail.

Definition 8: We call non-chaotic window of (3.3) the set of values of c for which there exists an exponentially stable non-chaotic solution of (3.3).

Definition 9: We call complete synchronization window (CSW) of (3.3), the set of values of c for which there exists a function $s(t)$ such that $(x(t), y(t))=(s(t), s(t))$ is an exponentially stable solution of (3.3).

Considering the previous definitions, zones 1 and 3 correspond to values of $c$ of the non-chaotic window, while zone 2 corresponds to values of the complete-synchronization window. Although zones 1 and 3 have similar aspects, they correspond to different forms of destruction of the chaotic behavior as can be verified by registering the iterations as a function of $\mathrm{t}$ for values of $\mathrm{c}$ in each one of the two windows. This distinct behavior is evidenced in the figure 3 . In this figure we show the graphs of $x$ and $y$ as functions of $t$ for $c=0.15$ (zone 1) and $c=0.85$ (zone 3 ): the subset of the non-chaotic window corresponding to zone 3 is related to a fixed point, whereas the subset of the non-chaotic window corresponding to zone 1 is related to a period-2-synchronizedout-of-phase behavior. It is useful to define two subsets of the non-chaotic window.

\subsubsection{Fixed Point Window and Period 2 - Synchronized Window}

Definition 10: We call fixed point window of (3.3) the set of values of cfor which there exist $x_{0}$ and $y_{0}$ such that $(x(t), y(t))=\left(x_{0}, y_{0}\right)$ is an exponentially stable solution of (3.3), i.e. the set of values of cfor which the coupling-c(3.3) has an exponentially stable fixed point ( $\left.x_{0}, y_{0}\right)$.

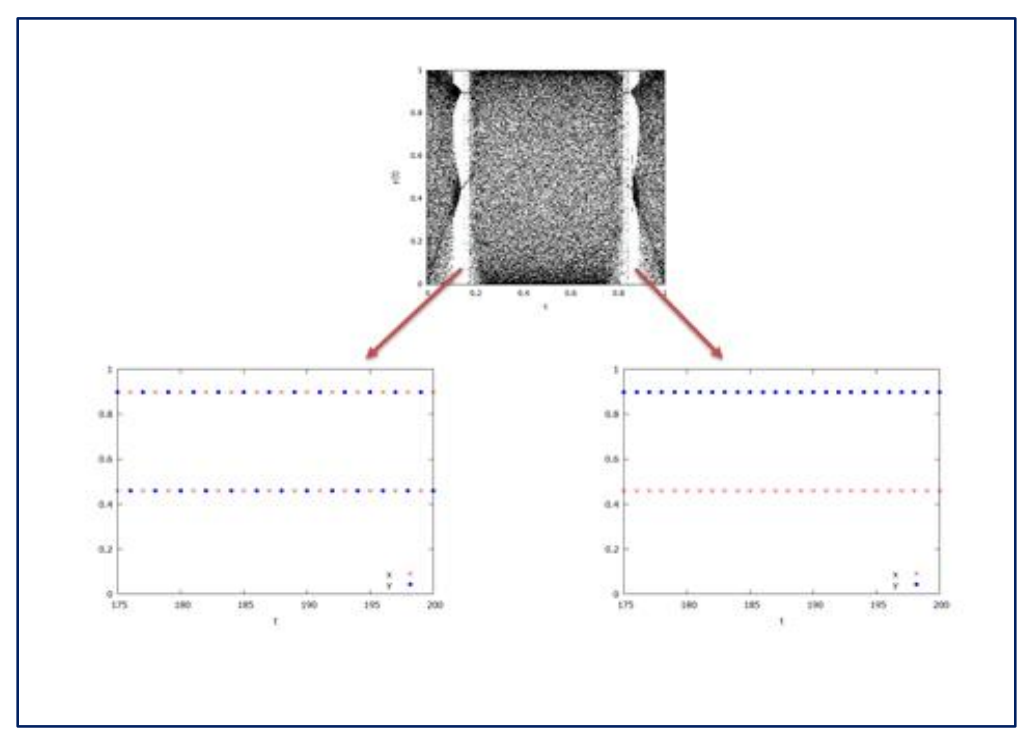

Fig. 3: Graphs of the iterations $x(t)$ and $y(t)$ as a function of $t$ for the symmetric linear coupling of the logistic with $c=0.15$ (left) and $c=0.85$ (right) 
According to the previous definition, zone 3 corresponds to a fixed point window.

Definition 11: We call period 2 - synchronized window of (3.3), the set of values of $c$ for which there exists an exponentially stable non-constant solution $(x(t+1), y(t+1))=(y(t), x(t))$ of (3.3), i.e. the set of values of $c$ for which the coupling-c (3.3) has an exponentially stable period-2 orbit $\left\{\left(x_{1}, x_{2}\right\},\left(x_{2}, x_{1}\right)\right\}\left(\right.$ with $\left.x_{1} \neq x_{2}\right)$.

According to the previous definition, zone 1 corresponds to a period 2 - synchronized window. It should be noted that the possibility of a non-empty non-chaotic window determines that coupling one chaotic dynamical system to another may be a strategy to suppress chaotic behavior (it will be when the non-chaotic window is not empty). It is, therefore, important to look at the fixed point window and the period2- synchronized window that we have found.

\subsubsection{Fixed point window}

We start by analyzing the fixed-point window, corresponding to zone 3 . For this purpose, we define a fixed-point line as one where the values of $\mathrm{x}$ and $\mathrm{y}$ correspond to the solutions of fixed point of the coupling. The fixed points $\left(x_{0}, y_{0}\right)$ of a Symmetric Linear Coupling correspond to the values $x_{0}$ and $y_{0}$ that satisfy

$$
\left\{\begin{array}{l}
x_{0}=(1-c) \cdot f\left(x_{0}\right)+c \cdot f\left(y_{0}\right) \\
y_{0}=c \cdot f\left(x_{0}\right)+(1-c) \cdot f\left(y_{0}\right.
\end{array}\right.
$$

This system of equations describes a line in the plane $x y$ that we designate by line-of-fixed-point of the Symmetrical Linear Coupling.

Definition 12: We call fixed-point line of (3.3) the line of the plane $x y$, which is described by the points $(x, y)=\left(x_{0}, y_{0}\right)$ such that

$$
\left\{\begin{array}{l}
x_{0}=f\left(x_{0}\right)+c \cdot\left[F_{1}\left(x_{0}\right)+F_{2}\left(y_{0}\right)\right] \\
y_{0}=g\left(y_{0}\right)+c \cdot\left[G_{1}\left(x_{0}\right)+G_{2}\left(y_{0}\right)\right]
\end{array}\right.
$$

or, equivalently, by

$$
\left(x_{0}-f\left(x_{0}\right)\right) \cdot\left[G_{1}\left(x_{0}\right)+G_{2}\left(y_{0}\right)\right]=\left(y_{0}-g\left(y_{0}\right)\right) \cdot\left[F_{1}\left(x_{0}\right)+F_{2}\left(y_{0}\right)\right]
$$

We designate by 3D-fixed-point line of coupling (3.3), the line of the space xyc described by (3.7).

By summing up the two equations of (3-6), the fixed-line description of the symmetric linear coupling takes the form

$$
x_{0}+y_{0}=f\left(x_{0}\right)+f\left(y_{0}\right)
$$


Only some of its points correspond to exponentially stable solutions.

Proposition 1: Considering a point $\left(x_{0}, y_{0}\right)$ of the fixed point-line of the Symmetric Linear Coupling (3.5), if the absolute values of both eigenvalues of matrix

$$
D J_{\left(x_{0}, y 0\right)}=\left[\begin{array}{cc}
(1-c) f^{\prime}\left(x_{0}\right) & c f^{\prime}\left(y_{0}\right) \\
c f^{\prime}\left(x_{0}\right) & (1-c) f^{\prime}\left(y_{0}\right)
\end{array}\right]
$$

with $c=\frac{x_{0}-f\left(x_{0}\right)}{f\left(y_{0}\right)-f\left(x_{0}\right)}$, are less than one, then $\left(x_{0}, y_{0}\right)$ is an exponentially fixed point of (3.5), i. e. c belongs to the fixed-point window of (3.5). If at least one of the eigenvalues of $D J\left(x_{0}, y_{0}\right)$ has absolute value greater than one, then $\left(x_{0}, y_{0}\right)$ is an unstable fixed point of (3.5), i.e. c does not belong to the fixed-point window of (3.5).

Applying the proposition to the Linear Symmetric Coupling of logistic and tent maps, we can identify which points of the fixed-point lines correspond to values of $c$ belonging to the fixedpoint window.

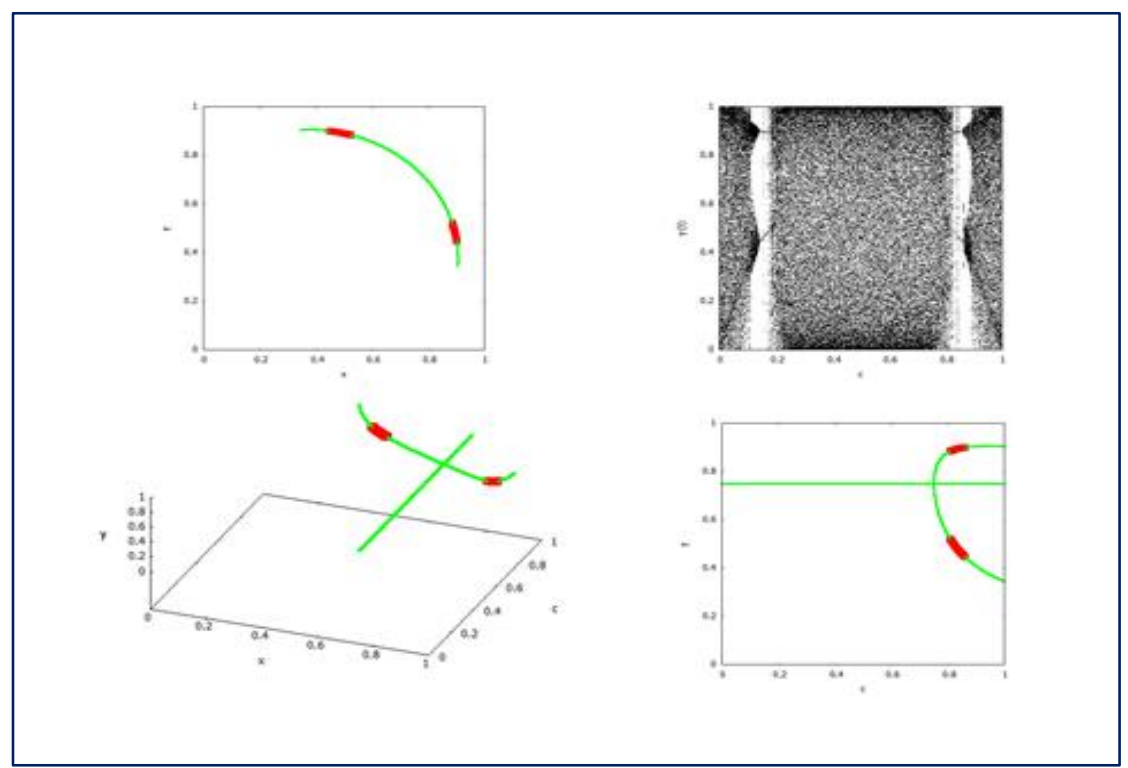

Fig. 4: Graph of iterations $x(t)$ as a function of $c$ for Symmetric Linear Coupling of logistic maps (top right) and corresponding fixed-point-line (top left), line-3D-of the fixed points (bottom left) and its projection onto xc plane (bottom right). The points corresponding to exponentially stable solutions are marked in red

In Fig. 4 and Fig. 5, the points corresponding to values of $c$ of the fixed-point window are shown in red, that corresponding to $|\lambda|<1$. When the dynamic of the free systems is described by a sectionally linear map, it is easy to obtain analytically the fixed-point window.

Proposition 2: The fixed-point window for the tent map is empty. 


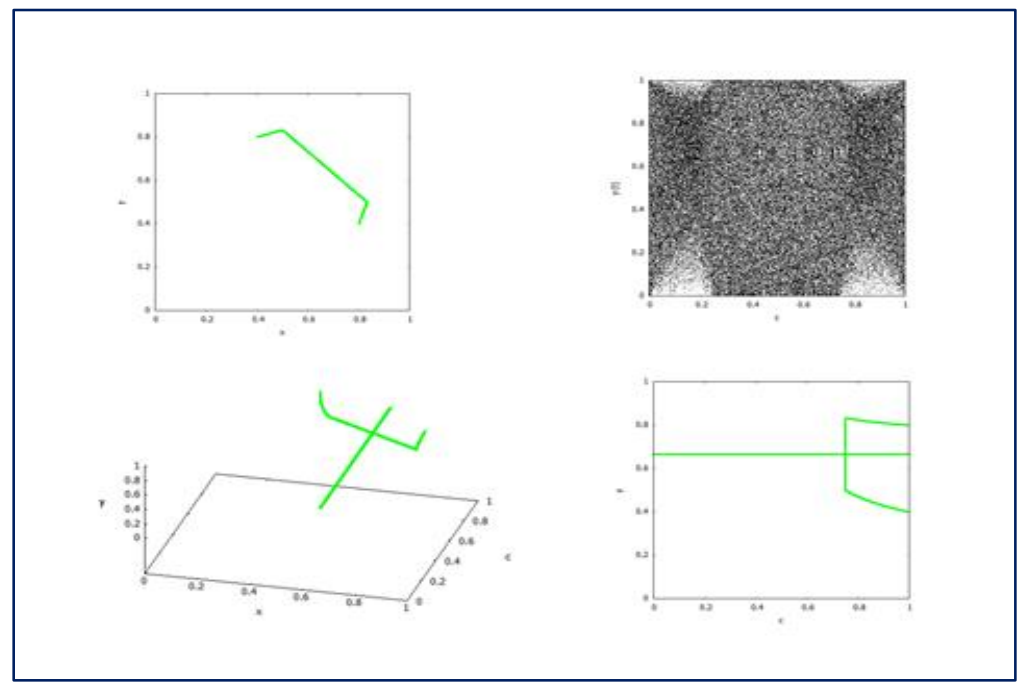

Fig. 5: Graph of the iterations $x(t)$ as a function of $c$ for the Symmetrical Linear Coupling of tent maps (top right) and the corresponding fixed-point line (top left), line-3D-of fixed points (bottom left) and its projection onto the xc plane (bottom right)

It will therefore be desirable to obtain a strategy to try to find out if a coupling has an empty or non-empty fixed-point window and to locate it when it is not empty. The following proposition provides a procedure to check whether the symmetric linear coupling of maps $f$ admits a nonempty fixed-point window.

Proposition 3: If $\left|(1-c) . f^{\prime}\left(y_{0}\right)\right|<1$ with $c=\frac{x_{0}-f\left(x_{0}\right)}{f\left(y_{0}\right)-f\left(x_{0}\right)}, x_{0}$ and $y_{0}$ such that $f^{\prime}\left(x_{0}\right)=0$ and $x_{0}+y_{0}=f\left(x_{0}\right)+f\left(y_{0}\right)$ then the Symmetric Linear Coupling of dynamical systems described by the map fadmits a non-empty fixed-point window.

We exemplify the use of this procedure, applying it to the case of the cubic map, following the strategy described in the proposition.

i) $\quad f^{\prime}\left(x_{0}\right)=0 \Leftrightarrow x_{0} \simeq 0.253$ or $x_{0} \simeq 0.747$

ii) for $x_{0}=0.253, x_{0}+y_{0}=f\left(x_{0}\right)+f\left(y_{0}\right)$ provides $y_{0} \simeq 0.747$, and for $x_{0}=$ $0.747, x_{0}+y_{0}=f\left(x_{0}\right)+f\left(y_{0}\right)$ provides $y_{0} \simeq 0.253$

iii) in both situations $\left(x_{0}=0.253\right.$ or $\left.x_{0}=0.747\right), c=\frac{x_{0}-f\left(x_{0}\right)}{f\left(y_{0}\right)-f\left(x_{0}\right)} \simeq 0.776$, to which corresponds $\left|(1-c) \cdot f^{\prime}\left(y_{0}\right)\right| \simeq 0.001<1$.

Fig. 6 summarizes the behavior of the Symmetric Linear Coupling for the cubic, as Figs. 4 and 5 summarized for $f_{L}$ and $f_{\mathrm{T}}$, respectively, and confirms the existence of a non-empty fixed-point window including the value of $c=0.776$ provided by the strategy used.

So, we conclude that the chaotic behavior of a dynamical system corresponding to the cubic map is destructible if we couple it (with the appropriate value of $c$ ) to an identical dynamic system using a symmetric linear coupling. 


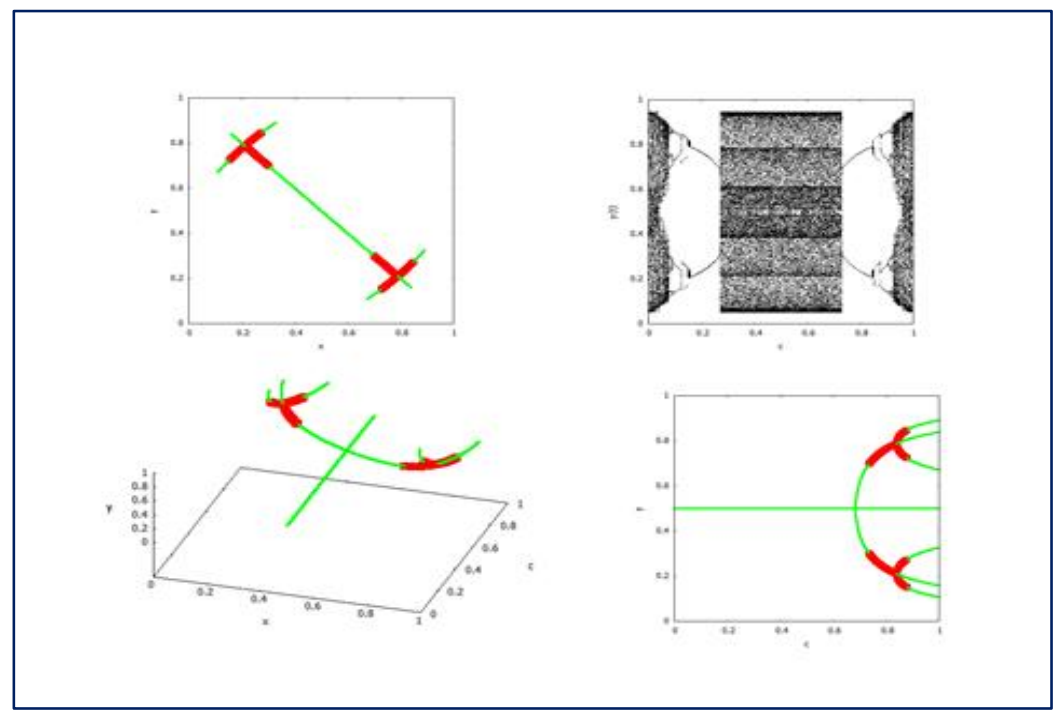

Fig. 6: Graph of the iterations $x(t)$ as a function of $c$ for the Symmetric Linear Coupling of cubic maps (top right) and the corresponding fixed-point line (top left), 3D-line of fixed points (bottom left) and its projection onto the xc plane (bottom right). The exponentially stable fixed points are marked in red

\subsubsection{Period 2 - Synchronized Window}

In this Section, we analyze the phase 2 synchronized window of a Symmetric Linear Coupling, corresponding to zone 1 . In order that a trajectory $\{(\alpha, \beta),(\beta, \alpha)\}$ is a solution of (3.5) the following four equations need to be satisfied:

$$
\begin{array}{r}
\left\{\begin{array}{l}
\beta=(1-c) \cdot f(\alpha)+c \cdot f(\beta) \\
\alpha=c \cdot f(\alpha)+(1-c) \cdot f(\beta)
\end{array}\right. \\
\left\{\begin{array}{l}
\alpha=(1-c) \cdot f(\beta)+c \cdot f(\alpha) \\
\beta=c \cdot f(\beta)+(1-c) \cdot f(\alpha)
\end{array}\right.
\end{array}
$$

Since the last two equations are exactly the same as the first ones, we introduce the following definition of line-of-period-2-synchronized-of a Symmetric Linear Coupling:

Definition 13: We call period-2-synchronized line of (3.5), the line of the plane $x y$ which is described by the points $(x, y)=(\alpha, \beta)$ such that

$$
\left\{\begin{array}{l}
\beta=(1-c) \cdot f(\alpha)+c \cdot f(\beta) \\
\alpha=c \cdot f(\alpha)+(1-c) \cdot f(\beta)
\end{array}\right.
$$

or, equivalently, by

$$
\alpha+\beta=f(\alpha)+f(\beta)
$$

We call period-2-3D-synchronized line of the coupling (3.5) the line of space xyc described by (3.10). 
A point $(\alpha, \beta)$ of the period-2-synchronized line only corresponds to a situation of suppression of the chaotic behavior, i.e. only corresponds to values of $c$ belonging to the period-2-synchronized window, if it is associated with an exponentially stable solution of (3.5). The following proposition determines which points of the period-2-synchronized line of a Symmetric Linear Coupling correspond to values of $c$ belonging to the window-of-period-2-synchronized

Proposition 4: Considering a point $(\alpha, \beta)$ of the period-2-synchronized line of the Symmetric Linear Coupling (3.5), if the absolute values of the eigenvalues of

$$
D J_{2}(\alpha, \beta)=\left[\begin{array}{cc}
(1-c) \cdot f^{\prime}(\beta) & c f^{\prime}(\alpha) \\
c f^{\prime}(\beta) & (1-c) \cdot f^{\prime}(\alpha)
\end{array}\right] \cdot\left[\begin{array}{cc}
(1-c) \cdot f^{\prime}(\alpha) & c f^{\prime}(\beta) \\
c f^{\prime}(\alpha) & (1-c) \cdot f^{\prime}(\beta)
\end{array}\right]
$$

with $c=\frac{\beta-f(\alpha)}{f(\beta)-f(\alpha)}$, are less than one, then $\{(\alpha, \beta),(\alpha, \beta)\}$ is a trajectory exponentially stable of (3.5), i.e. $c$ belongs to the period-2-synchronized window of (3.5). If at least one of the eigenvalues of $D J_{2}(\alpha, \beta)$ has an absolute value greater than one, then $\{(\alpha, \beta),(\alpha, \beta)\}$ is an unstable trajectory of (3.5), i.e. c does not belong to the period-2-sync window of (3.5).

Applying this proposition to the Symmetric Linear Coupling of the maps that we used in subsection 3.1.2.1, i.e., applying this proposition to the maps of the logistic and the tent, we can identify what are the points of its period-2-synchronized line that correspond to values of $c$ belonging to the period-2-synchronized window. In Figs. 7 and 8 we present the period-2synchronized lines for the two couplings, marking in red the points corresponding to exponentially stable solutions $\{(\alpha, \beta),(\alpha, \beta)\}$.

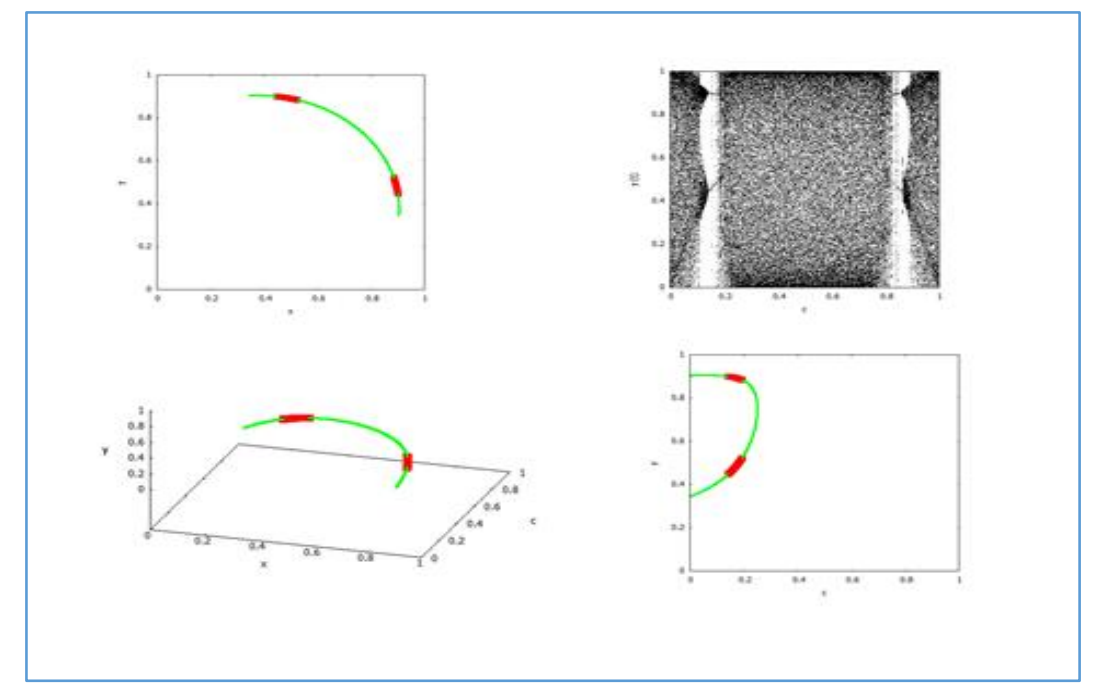

Fig. 7: Graph of iterations $x(t)$ as a function of $c$ for Symmetrical Linear Coupling of logistic maps (top right) and corresponding period-2-synchronized line (top left), 3D- 2-synchronized (bottom left) and its projection onto plane $x c$ (bottom right). The points corresponding to exponentially stable solutions are marked in red 
These results confirm those shown in Fig. 2 in relation to the period-2-synchronized window, namely that the period-2-synchronized window of the Symmetric Linear Coupling of logistic maps is not empty. The same is confirmed for the tent map by obtaining that the period-2synchronized window of the Symmetric Linear Coupling of tent maps is empty. In addition, period-2-synchronized lines are exactly the same as the corresponding fixed-point lines, as we noted earlier. However, while the fixed-point window of the Symmetric Linear Coupling is ]0.806, $0.861[$ its period-2-synchronized window is approximately ]0.139, $0.194[$.

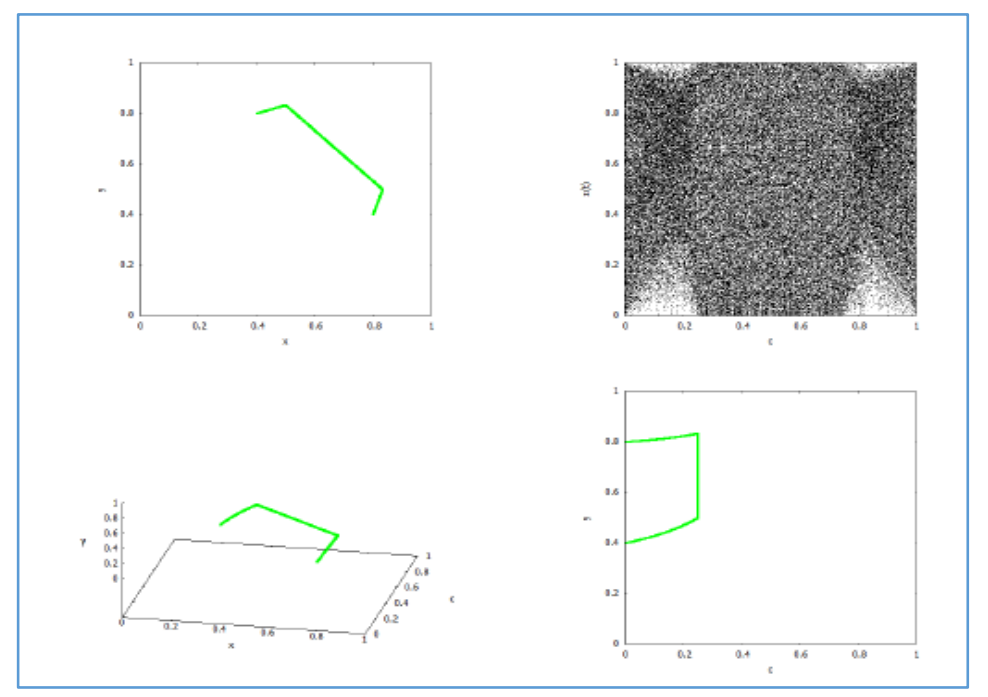

Fig. 8: Graph of iterations $x(t)$ as a function of $c$ for Symmetrical Linear Coupling of tent maps (top right) and corresponding period-2-synchronized line (top left), 3D-period-2-synchronized (bottom left) and its projection onto the plane $x c$ (bottom right)

\subsubsection{Complete-Synchronization Window}

In this section we analyze the situation designated by zone 2 in Fig. 2 and that we identified as corresponding to the complete synchronization window.

Note that for $c=1 / 2$ the coupling (3.5) is reduced to

$$
\left\{\begin{array}{l}
x(t+1)=\frac{1}{2} f(x(t))+\frac{1}{2} \cdot f(y(t)) \\
y(t+1)=\frac{1}{2} \cdot f(x(t))+\frac{1}{2} \cdot f(y(t))
\end{array}\right.
$$

The coupled dynamic systems completely synchronize after the first iteration. So the complete synchronization window of the Symmetric Linear Coupling is always non-empty, whatever the free dynamics of the coupled systems are, i.e. whatever is $f$, the window will always contain $c=$ 1/2. In the graphs of the iterations $y(t)-x(t)$ as a function of $c$, the values of $c$ that belong to the complete-synchronization window correspond to ones that its images are null (since for such values of $c, y(t) \simeq x(t)$, for sufficiently large values of $t$ ). In Fig. 9, we present these graphs for Symmetric Linear Coupling of the four free dynamics that have been considered: logistic, tent, 
cubic and saw. We verify that complete synchronization happens not only for $c=1 / 2$ but also other values in a set centered on $c=1 / 2$.

In a Symmetric Linear Coupling, whatever the coupling constant, $c$, is, the system of equations (3.5) admits the solution $(x(t), y(t))=(s(t), s(t))$, where $s(t)$ is a function that satisfies $s(t)=$ $f(s(t))$. However, a given value of $c$ will only belong to the complete-synchronization window if $(x(t), y(t))=(s(t), s(t))$ is an exponentially stable solution. The complete-synchronized window can be determined analytically, as the following result states:

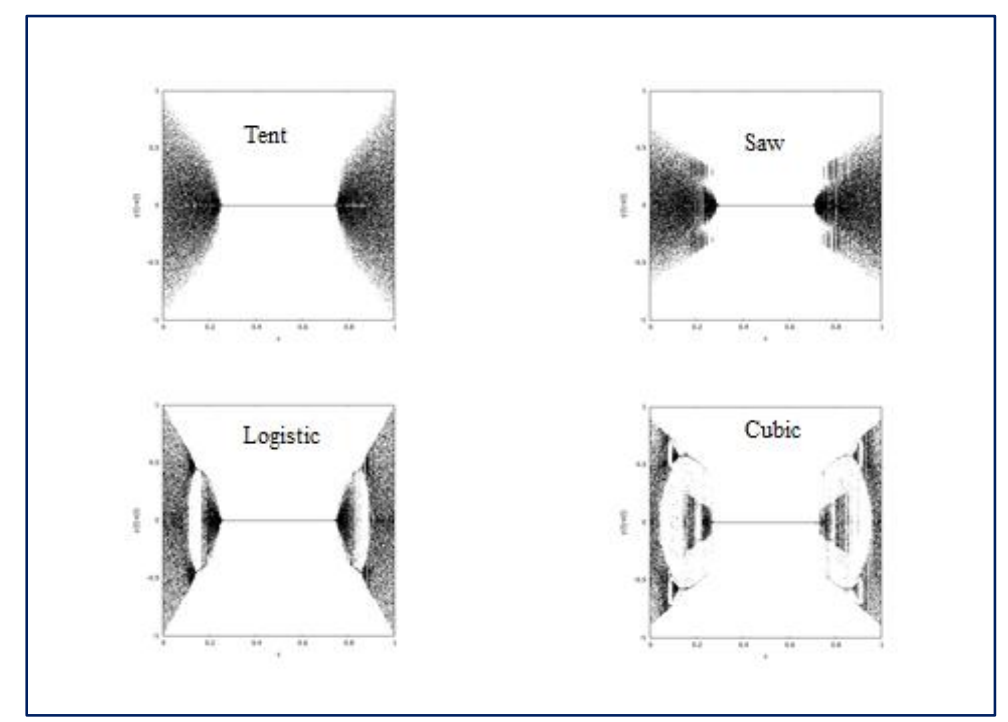

Fig. 9: Graphs of the iterations $y(t)-x(t)$ as a function of c for the Symmetrical Linear Coupling

Proposition 5: The complete-synchronization window of a Symmetric Linear Coupling (3.5) is,

$$
\operatorname{CSW}=] \frac{1-e^{-h}}{2}, \frac{1+e^{-h}}{2}[
$$

where $h$ is the Lyapunov exponent corresponding to the map $f$.

Taking into account the values of the Lyapunov exponents of logistics, tent, cubic and saw maps obtained in section $2.2(\ln 2, \ln 2, \ln 2.4$ and 0.715 , respectively), the above proposition provides the following complete-synchronization windows for these maps:

Tent and logistic: $C S W=]$ 0.25, 0.75 [

Saw: $\operatorname{CSW}=] 0.291(6), 0.708(3)[$

Cubic: $\operatorname{CS} W \simeq] 0.25571,0.74429$ [

These results coincide with those obtained using the numerical approach that provided the graphs of Fig. 9. So, the basins of attraction of the exponentially stable completely synchronized solutions are large enough that a random choice of initial iterations does not mask the completesynchronized window. 


\subsection{Other Linear Couplings}

We will now extend some of the results obtained in the previous subsection 3.1 to other couplings of the type (3.3).

\subsubsection{Fixed-point windows in other couplings}

Considering a coupling (3.3), each point $\left(x_{0}, y_{0}\right)$ of its fixed-point line (3.7) corresponds to a value of the coupling constant, namely a

$$
c=\frac{x_{0}-f\left(x_{0}\right)}{F_{1}\left(x_{0}\right)+F_{2}\left(y_{0}\right)}=\frac{y_{0}-g\left(y_{0}\right)}{G_{1}\left(x_{0}\right)+G_{2}\left(y_{0}\right)}
$$

As for Symmetric Linear Coupling, and according to the next proposition, only a few points of this fixed-point line or even none at all, correspond to values of $c$ belonging to the fixed-point window of (3.3).

Proposition 6: Considering a point ( $\left.x_{0}, y_{0}\right)$ of the fixed-point line (3.5), if the absolute values of both eigenvalues of

$$
D J_{(x 0, y 0)}=\left[\begin{array}{cc}
f^{\prime}\left(x_{0}\right)+c \cdot F_{1}{ }^{\prime}\left(x_{0}\right) & c . F_{2}{ }^{\prime}\left(y_{0}\right) \\
c . G_{1}{ }^{\prime}\left(x_{0}\right) & g^{\prime}\left(y_{0}\right)+c \cdot G_{2}{ }^{\prime}\left(y_{0}\right)
\end{array}\right]
$$

with $c=\frac{x_{0}-f\left(x_{0}\right)}{F_{1}\left(x_{0}\right)+F_{2}\left(x_{0}\right)}$, are smaller than one, then $\left(x_{0}, y_{0}\right)$ is an exponentially stable fixed point, i.e. c belongs to the fixed-point window of (3.3). If at least one of the eigenvalues of $D J\left(x_{0}, y_{0}\right)$ has absolute value larger than one, then $\left(x_{0}, y_{0}\right)$ is an unstable fixed point and cdoes not belong to the fixed-point window of (3.3).

In couplings such that $F_{1}=-G_{1}$ and $F_{2}=-G_{2}$ the fixed-point line (3.8) reduces to $x_{0}+y_{0}=$ $f\left(x_{0}\right)+g\left(y_{0}\right)$. This is, for example, the case in Linear Couplings. In Linear Couplings of nonidentical dynamical systems, the expression of the eigenvalues of DJ, is given by

$$
\lambda=\frac{1}{2}(1-c) \cdot\left(f^{\prime}\left(x_{0}\right)+g^{\prime}\left(y_{0}\right)\right) \pm \frac{1}{2} \sqrt{(1-c)^{2} \cdot\left(f^{\prime}\left(x_{0}\right)+g^{\prime}\left(y_{0}\right)\right)^{2}-4(1-2 c) \cdot f^{\prime}\left(x_{0}\right) \cdot g^{\prime}\left(y_{0}\right)}
$$

Considering the previous proposition, that $f^{\prime}\left(x_{0}\right)$ (or $g^{\prime}\left(y_{0}\right)$ ) being zero determines that one of the eigenvalues is zero - the other eigenvalue being given by $\lambda=(1-c) \cdot g^{\prime}\left(y_{0}\right)$ - and using a strategy similar to the one we used for the Symmetric Linear Coupling, we obtain for the Linear Coupling of non-identical dynamical systems a way to determine if the fixed-point window is non-empty and to locate it. Using this strategy, we have calculated and located the fixed-point windows for Linear Couplings of a dynamical system described by the logistic map with another that is not identical to it, namely with another dynamical systems that is described by the tent map, the saw map and the cubic map.

(i) We detail the calculations in the case of coupling the logistics with the tent, that is, the case where $f=f_{L}$ and $g=f_{T}$ : 
$1-f^{\prime}\left(x_{0}\right)=0 \Leftrightarrow x_{0}=0.5$

2 - $\quad x_{0}+y_{0}=f_{L}\left(x_{0}\right)+f_{T}\left(y_{0}\right) \Leftrightarrow y_{0}=5 / 6$

3 - $\quad c=0.75$, to which corresponds $\left|(1-c) \cdot f^{\prime} T\left(y_{0}\right)\right|=0.5<1$.

We conclude that the chaotic behavior of the logistic is destructible if we couple it (with the appropriate value of $c$ ) with the tent, using a Linear Coupling. In Fig. 10, we present the fixedpoint-line of this coupling, marking in blue the points that correspond to exponentially stable solutions. We can verify that the fixed point that the proposed strategy provides, namely $\left(x_{0}, y_{0}\right)$ $=(1 / 2,5 / 6)$, is an exponentially stable fixed point, corresponding to a value of $c$, namely $c=0.75$, which belongs to the fixed-point window. In this figure we present the graph of the iterations $y$ $(t)$ as a function of $c$, which is obtained following the numerical approach proposed in subsection 3.1.1 and where the fixed-point window is also visible.

For the other referred couplings we present their graphs.

(ii) The case of coupling the logistic map with the saw map, that is, the case where $f=f_{L}$ and $g=f_{S}$ :

The existence of blue-marked points, corresponding to exponentially stable fixed points, means that the fixed-point window is not empty. The graph of iterations $y(t)$ as a function of $c$, which we present in Fig. 11, also shows this. Although the application to this coupling of the defined strategy fails to determine the existence and location of a non-empty fixed-point window, we conclude that the chaotic behavior of the logistic map is also destructible if we couple it (with the appropriate value of $c$ ) with the saw map using a Linear Coupling.

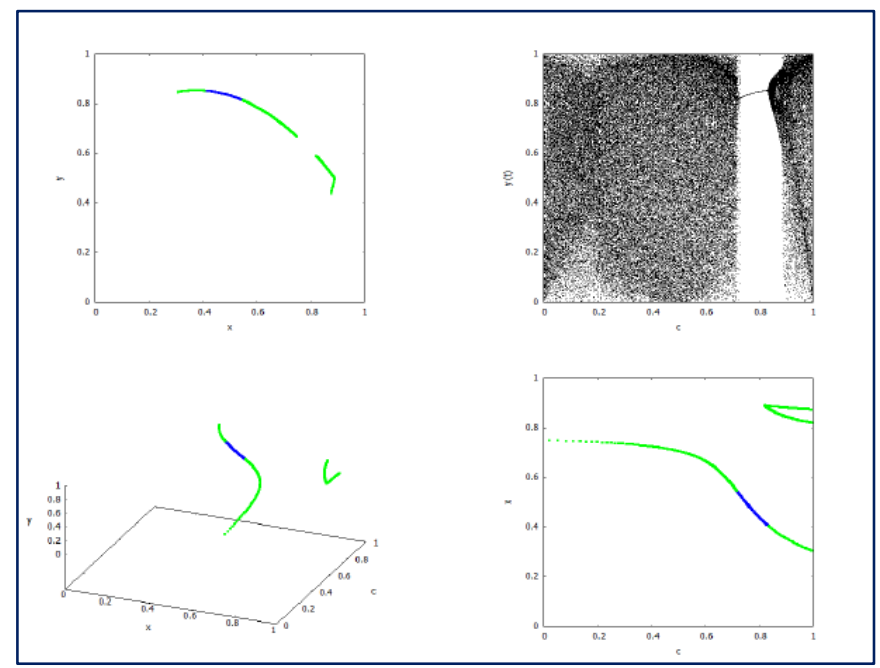

Fig. 10: Graph of the iterations $y(t)$ as a function of $c$ for the Linear Coupling of the logistic map with the tent map (top right) and corresponding fixed-point line (top left), 3D- fixed point line (bottom left) and its projection onto plane $x c$ (bottom right).

The exponentially stable fixed points are marked in blue 


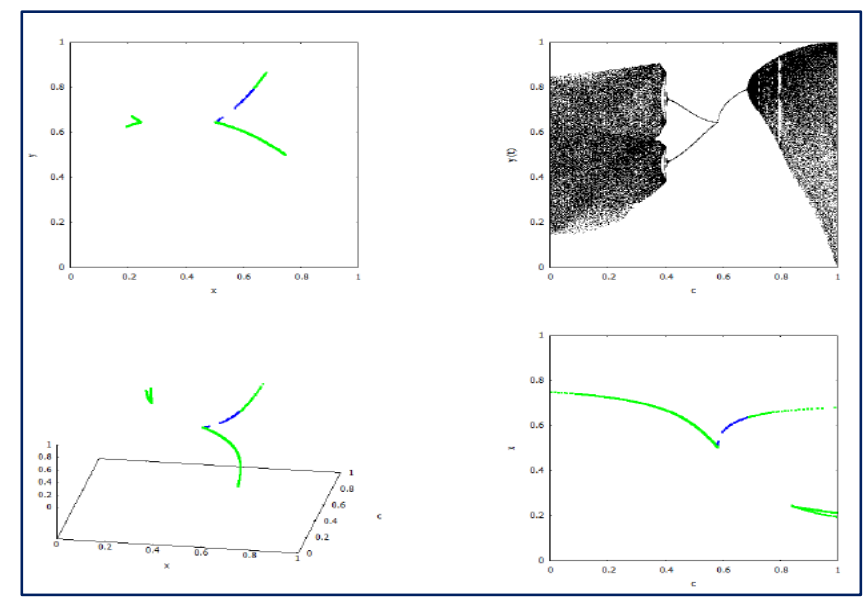

Fig. 11: Graph of iterations $y(t)$ as a function of $c$ for the Linear coupling of the logistic map with saw map (top right) and corresponding fixed-point line (top left), 3D-fixed-point line (bottom left) \& its projection onto plane $x c$ (bottom right). The exponentially stable fixed points are marked in blue

(iii) The case of coupling the logistic map with cubic map, i.e., the case where $f=f_{L}$ and $g=f_{c}$.

We conclude that the chaotic behavior of the logistic is destructible if we couple it (with the appropriate value of $c$ ) to the cubic, using a Linear Coupling. In Fig. 12, we present the fixedpoint line of this coupling, marking in blue those that are exponentially stable. We can verify that the fixed points proposed by the proposed strategy, namely $\left(x_{0}, y_{0}\right)=(0.5,0.647)$ and $\left(x_{0}, y_{0}\right)=(0.5$, 0.910 ), are exponentially stable fixed points, corresponding to values of $c$, namely $c \simeq 0.586$ and $c \simeq 0.847$, which belong to the fixed-point window. We present the graph of the iterations $y(t)$ as a function of $c$ (Fig. 12), which is obtained following numerical approach proposed in subsection 3.1.1, where fixed-point window is also visible.

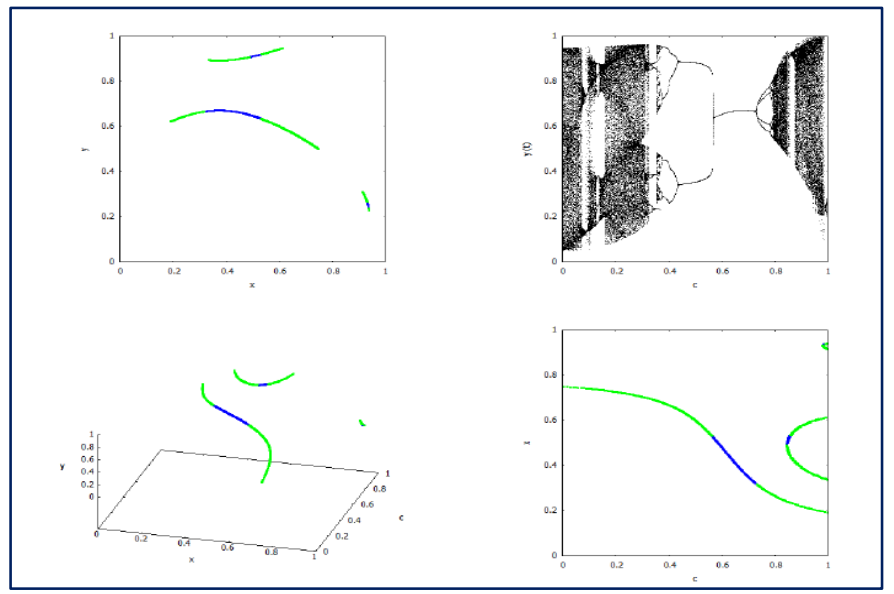

Fig. 12: Graph of the iterations $y(t)$ as a function of $c$ for the Linear Coupling of the logistic map with the cubic map (top right) and the corresponding fixed-point-line (top left), 3D- fixed point line (bottom left) and its projection onto the plane $x c$ (bottom right).

The exponentially stable fixed points are marked in blue 


\subsubsection{Period 2-synchronized Window}

The generalization for a coupling (3.3) of Definition 13 of a period-2 synchronized line corresponds to the points $(\alpha, \beta)$ that verify

$$
\begin{array}{r}
\left\{\begin{array}{l}
\beta=f(\alpha)+c \cdot\left[F_{1}(\alpha)+F_{2}(\beta)\right] \\
\alpha=g(\beta)+c \cdot\left[G_{1}(\alpha)+G_{2}(\beta)\right]
\end{array}\right. \\
\left\{\begin{array}{l}
\alpha=f(\beta)+c \cdot\left[F_{1}(\beta)+F_{2}(\alpha)\right] \\
\beta=g(\alpha)+c .\left[G_{1}(\beta)+G_{2}(\alpha)\right]
\end{array}\right.
\end{array}
$$

These are the equations that have to be satisfied so that a 2 -synchronized period trajectory $\{(\alpha, \beta),(\beta, \alpha)\}$ is solution of (3.3). Contrary to what happens for Symmetric Linear Coupling, the system of the four equations may have no solution, the period-2-synchronized line may be the empty set. However, if $f, g, F_{1}, F_{2}, G_{1}$ and $G_{2}$ meet certain conditions, a non-empty period-2synchronized line can be guaranteed.

If $f=g, F_{1}=G_{2}$ and $F_{2}=G_{1}$, that is, for couplings of following the type

$$
\left\{\begin{array}{l}
x(t+1)=f(x(t))+c \cdot\left[F_{1}(x(t))+F_{2}(y(t))\right] \\
y(t+1)=f(y(t))+c \cdot\left[F_{1}(x(t))+F_{2}(y(t))\right],
\end{array}\right.
$$

only two out of the four equations of (3.13) are linearly independent.

Definition 14: We call period-2-synchronized line of the coupling (3.14), the line of the plane xy which is described by the points $(x, y)=(\alpha, \beta)$ such that:

$$
\left\{\begin{array}{l}
\beta=f(\alpha)+c \cdot\left[F_{1}(\alpha)+F_{2}(\beta)\right] \\
\alpha=f(\beta)+c \cdot\left[F_{2}(\alpha)+F_{1}(\beta)\right]
\end{array}\right.
$$

We call 3D-period-2-synchronized-line of the coupling (3.13), the line of space xyc described by (3.14).

Contrary to what happens for a Symmetric Linear Coupling, the period-2-synchronized line of (3.14) is not equal to its fixed-point line, but all the same only some, or even none, of the period2-synchronized solutions correspond to exponentially stable solutions, that is, only some of them correspond to values of $c$ belonging to the period-2-synchronized window.

Proposition 7: Considering a point $(\alpha, \beta)$ of the period-2-synchronized line of the coupling (3.12), if the absolute of the eigenvalues of

$$
D J_{2}(\alpha, \beta)=\left[\begin{array}{cc}
f^{\prime}(\beta)+c . F_{1}{ }^{\prime}(\beta) & c . F_{2}{ }^{\prime}(\alpha) \\
c . F_{2}{ }^{\prime}(\beta) & f^{\prime}(\alpha)+c . F_{1}{ }^{\prime}(\alpha)
\end{array}\right] \cdot\left[\begin{array}{cc}
f^{\prime}(\alpha)+c . F_{1}{ }^{\prime}(\alpha) & c . F_{2}{ }^{\prime}(\beta) \\
c . F_{2}{ }^{\prime}(\alpha) & f^{\prime}(\beta)+c \cdot F_{1}{ }^{\prime}(\beta)
\end{array}\right]
$$

with $c=\frac{\beta-f(\alpha)}{F_{1}(\alpha)-F_{2}(\beta)}$, are less than one, then $\{(\alpha, \beta),(\alpha, \beta)\}$ is a trajectory exponentially stable of (3.14), that is, $c$ belongs to the period-2-synchronized window of (3.14). If at least one of the eigenvalues of $D J_{2}(\alpha, \beta)$ has an absolute value greater than one, then $\{(\alpha, \beta),(\alpha, \beta)\}$ is an unstable trajectory of (3.14) \& $c$ does not belong to the period-2-synchronized window of (3.14). 
The Symmetrical Linear Coupling is the only Linear Coupling of type (3.14) (corresponds to the choice $F 2=-F 1=f$ ). We introduce another coupling of type (3.14), one that we call Past Symmetric Coupling corresponding to choose $F 1=-f$ and $F 2=i d$.

$$
\left\{\begin{array}{l}
x(t+1)=(1-c) f(x(t))+c \cdot y(t) \\
y(t+1)=c \cdot x(t)+(1-c) f(y(t))
\end{array}\right.
$$

The period-2-synchronized line of a Past Symmetric Coupling is just $\left\{\left(\gamma_{1}, \gamma_{2}\right),\left(\gamma_{2}, \gamma_{1}\right)\right\}$, where $\gamma_{1}$ and $\gamma_{2}$ are the values corresponding to the period-2 trajectory $\left\{\gamma_{1}, \gamma_{2}\right\}$ of the free dynamical systems $x(t+1)=f(x(t))$.

(i) Past Symmetric coupling for the logistic map:

The values $\gamma_{1}$ and $\gamma_{2}$ of the period 2 trajectory $\left\{\gamma_{1}, \gamma_{2}\right\}$ of the logistic map are $\gamma_{1} \simeq 0.345$ and $\gamma_{2}$ $\simeq 0.904$, corresponding to

$$
D J 2(\gamma 1, \gamma 2) \simeq\left[\begin{array}{cc}
-3 c^{2}+8 c-4 & -6.472 . c(1-c) \\
2.472 . c(1-c) & -3 c^{2}+8 c-4
\end{array}\right]
$$

whose absolute values of the eigenvalues are less than one for values of coupling constant $c$ greater than $c \simeq 0.600$, that is, the period 2-synchronized window is approximately ] 0.600,1[.

(ii) Past Symmetric Coupling Past for the tent map

The period 2 trajectory of the tent map is $\{2 / 5,4 / 5\}$, corresponding to

$$
D J 2(2 / 5,4 / 5) \simeq\left[\begin{array}{cc}
-3 c^{2}+8 c-4 & 4 c^{2}-4 c \\
-4 c^{2}+-4 c & -3 c^{2}+8 c-4
\end{array}\right]
$$

whose absolute values of the eigenvalues are less than one for values of coupling constant $c$ greater than $c \simeq 0.600$, that is, the period 2 -synchronized window is approximately ] $0.600,1$. The results of the numerical approach are shown in figure 13, where we present the graphs of the $x(t)$ iterations as a function of $c$ exhibiting the period-2-synchronized windows that we just obtained analytically.

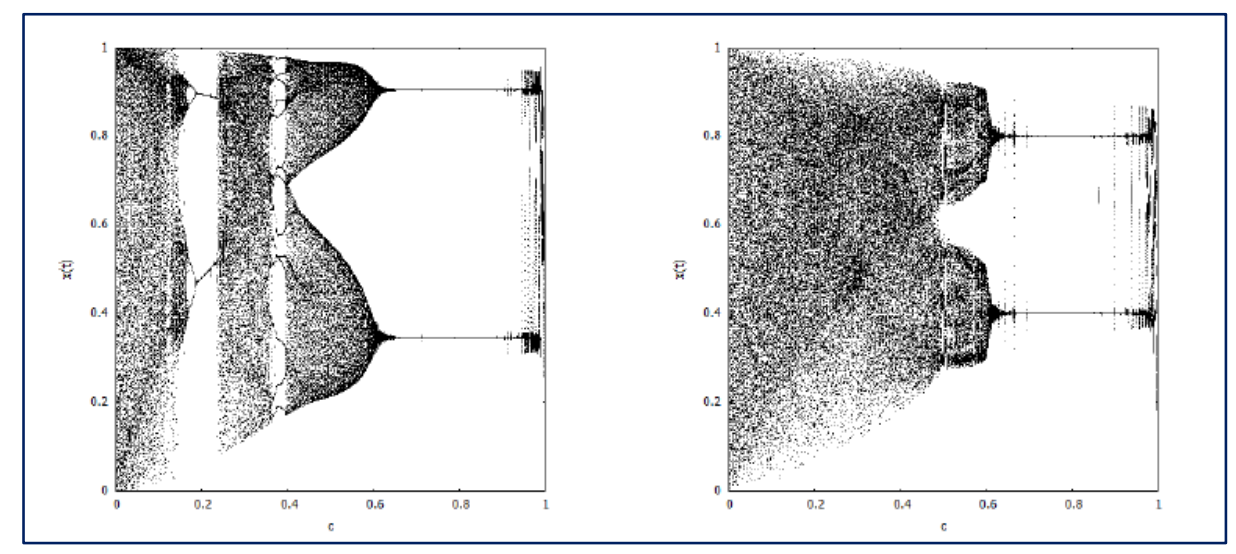

Fig. 13: Graphs of iterations $x(t)$ as a function of c for the Past Symmetric Coupling of the logistic map (left) and tent map (right) 


\section{References}

[1] Aulbach B and Kieninger B (2001), On Three Definitions of Chaos, Nonlinear Dynamics and Systems Theory, 1(1): 23-37.

[2] Blaya A and López V (2012), On the relations between positive Lyapunov exponents, positive entropy, and sensitivity for interval maps, Discrete and Continuous Dynamical Systems, 32(2): 433-466.

[3] Chen $\mathrm{T}$ and Zhu Z (2007), Exponential synchronization of nonlinear coupled dynamical networks, International Journal of Bifurcation and Chaos, 17(3): 999-1005.

[4] Devaney R (1989), An Introduction to Chaotic Dynamical Systems, Addison- Wesley Publishing Company.

[5] Ding M and Yang W (1997), Stability of synchronous chaos and on-off intermittency in coupled map lattices, Phys. Rev. E, 56(4): 4009-4016.

[6] Foucart S (2001), On definitions of discrete topological chaos and their relations on intervals, Cambridge Tripos Part III Essay.

[7] Li X and G Chen (2003), Synchronization and Desynchronization of Complex Dynamical Networks: An Engineering Viewpoint, IEEE Trans. Circuits Syst. I, 50(11): 1381-1390.

[8] Lopes L, Fernandes S and Grácio C (2014), Complete synchronization and delayed synchronization in couplings, Nonlinear Dynamics, 76(3): Springer.

[9] Lopes L, Ramos C, Fernandes S, Thapa GB and Grácio C (2018), Discrete Dynamical Systems: A Brief Survey, Journal of the Institute of Engineering, 14(1): 35-51.

[10] Fournier-Prunaret D, Rocha J, Caneco A, Fernandes S and Gracio C (2013), Synchronization and basins of synchronized states 2-dimensional piecewise maps issued of coupling between 3-pieces one-dimensional maps, Int. J. Bifurcation Chaos 23.

[11] Qiu J, Lu J, Cao J and He H (2011), Tracking analysis for general linearly coupled dynamical systems, Communications in Nonlinear Science and Numerical Simulation, 16: 2072-2085.

[12] Rangarajan G and Ding M (2002), Stability of Synchronized Chaos in Coupled Dynamical Systems, Phys. Lett. A, 296: 204-209. 